\title{
Grid Based on the Sierpinski Fractal and an Assessment of the Prospects for its Application in Aircraft Parts
}

\author{
Leviin Zhikharev ${ }^{1}$ \\ ${ }^{1}$ Moscow Aviation Institute (National Research University), Volokolamsk highway, 4, Moscow, 125993, Russia
}

\begin{abstract}
Modern geometric methods open up prospects for improving the shape and structure of parts. Such improvement can pursue the goals of increasing the strength with constant material consumption, or reducing the mass when it is not necessary to increase the strength. The meaning of geometric methods is to create a part shape the stresses arising in the part material under the action of applied loads are distributed most evenly. Such methods include the use of fractal geometry. This article presents the results of a study of a fractal lattice created on the basis of the Sierpinski triangle. Computer simulation in the SolidWorks, as well as strength studies of parts produced using additive technologies, allowed us to confirm a multiple increase in the strength of the fractal lattice with an increase in the number of fractal iterations. One of the most promising areas of application of fractal structures may be aviation technology. In this area, weight reduction is needful, and the complex shape of the parts is realized with the help of expensive production methods. For this reason, a number of experiments were conducted within the framework of the study, the purpose of which was to test the feasibility of using fractal gratings to reduce the weight of aircraft parts, using the example of the fork of the front landing gear of the combat training aircraft Yak-130.
\end{abstract}

\section{Keywords}

Fractal structures, Sierpinski grid, Yak-130, chassis fork

\section{Introduction}

Compliance with strict mass and strength requirements is the problem of creating aircraft structures. Special mass restrictions is the specifics of this field of design. Non-compliance with mass requirements can lead to increased fuel consumption and violation of the aerodynamic characteristics of the aircraft and even to its complete inactivity. The reduction in the mass of the device due to the weight loss of its individual parts and assemblies, which occurs without loss of strength, can have a positive effect on its operational characteristics, such as load capacity, efficiency and flight range. In this regard, it is relevant to develop ways to reduce the weight of aircraft parts $[1,2]$.

In modern science, there are many ways to reduce the mass of the power elements of parts by increasing their strength, which allows you to reduce the amount of material consumed. These include the use of new materials and composites $[3,4,5]$, the improvement of production methods and surface treatment of products, as well as the rationalization of the geometry of parts and the structure of their material. Geometric methods of reducing the mass of structures often lead to the formation of a complex shape that requires modern methods of their manufacture, most of which are associated with additive manufacturing technology $[2,6]$.

In addition to the use of rational cross-sections, truss, lattice structures and topology optimization [7], the use of fractal structures [8,9] belongs to the geometric method. In the article [10], the effectiveness of increasing the strength of light structures using fractal geometry created on the basis of the Sierpinski triangle was theoretically confirmed.

GraphiCon 2021: 31st International Conference on Computer Graphics and Vision, September 27-30, 2021, Nizhny Novgorod, Russia EMAIL: Zhabafrog@mail.ru (L. A. Zhikharev) ORCID: 0000-0001-8905-5101 (L. A. Zhikharev)

(c) (i) (C) 2021 Copyright for this paper by its authors.

Use permitted under Creative Commons License Attribution 4.0 International (CC BY 4.0).

CEUR Workshop Proceedings (CEUR-WS.org) 
Within the framework of this study, the problem of evaluating the possibility of using such structures to reduce the weight of aircraft parts is solved on the example of the chassis element of the Yak-130 military training aircraft.

\section{Theoretical foundations}

The geometric methods mentioned above do not allow us to directly solve the problem of increasing the strength, defined as the ability of a material not to collapse under the influence of a load until a critical stress value is reached. The essence of these methods is to rationalize the shape, which allows you to evenly distribute the stress throughout the material of the part. Thus, when bending a beam, the stress is distributed unevenly across its cross-section: the most stretched and compressed areas on the periphery of the section, as far as possible from the neutral plane, while with central compression and tension, the stress can be considered evenly distributed throughout the cross-section with certain assumptions. It follows from this that the replacement of the work of the elements of the part for bending and torsion to tension and compression is promising. This technique is used in the design of suspension bridges (Fig. 1). In cases where such a replacement is impossible, when designing elements that work on bending, rational sections are used, such as hollow pipes, I-beams, and others.

When solving the problem of reducing the mass of

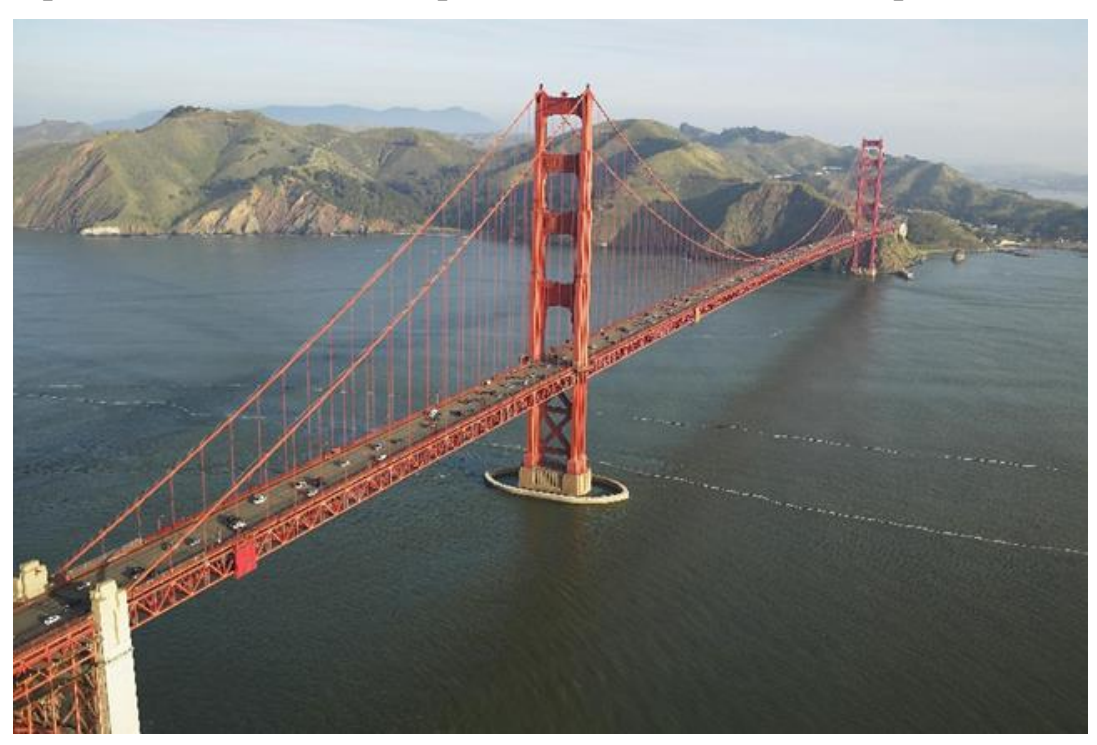

Figure 1: The example of replacing bending with stretching and clamping is suspension bridge

structural elements working on compression, reducing their cross-sectional area leads to the fact that such elements begin to collapse not due to excessive stress in the cross-section, but due to buckling. The critical load at the central compression of a thin rod is described by the Euler formula:

$$
\mathrm{P}_{\mathrm{b}}=\pi \mathrm{EJ} \times(\mathrm{KL})^{-2},
$$

where $\mathrm{E}$ is Young's modulus of the rod material, $\mathrm{J}$ is the minimum area moment of inertia of the crosssection, $\mathrm{L}$ is the unsupported length of the rod, and $\mathrm{K}$ is rod effective length factor. In accordance with this expression, reducing the effective length of the rod by two times increases the buckling stability of the rod by four times. This is the main idea of using the Sierpinski triangle, since in each new iteration of the fractal, the effective length of all its elements is reduced by two (Fig. 2).
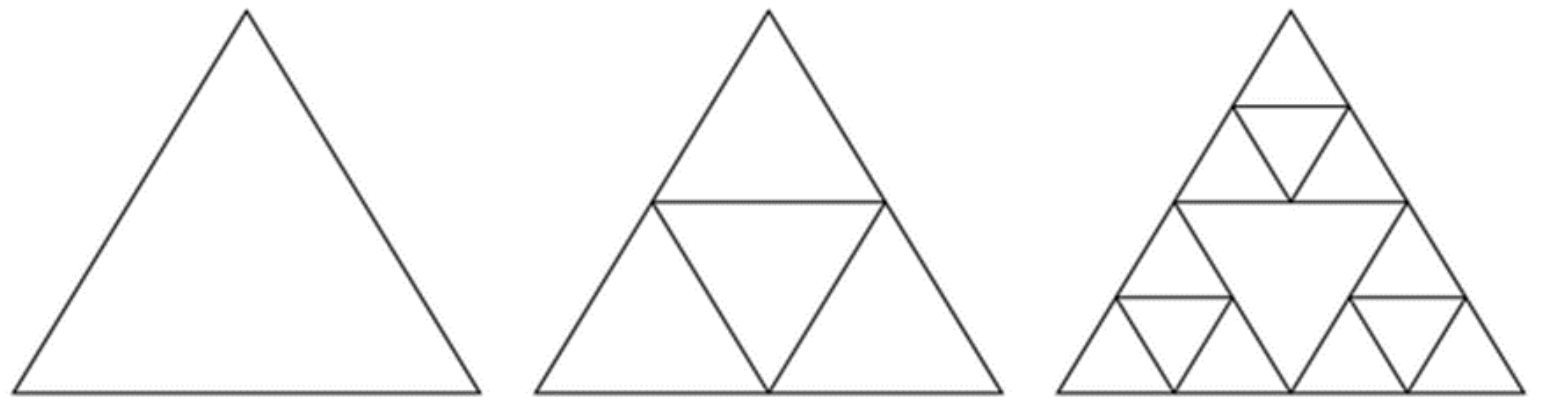

Figure 2: The first three iterations of constructing a Sierpinski triangle [10] 


\section{The experimental}

In the power elements of aircraft parts, an I-beam cross-section is often used. As part of this study, the fork of the front landing gear of the Russian military training aircraft Yak-130 was considered as an example [11]. The model, created from open sources-drawings and photographs, also contains similar elements (Fig. 3).

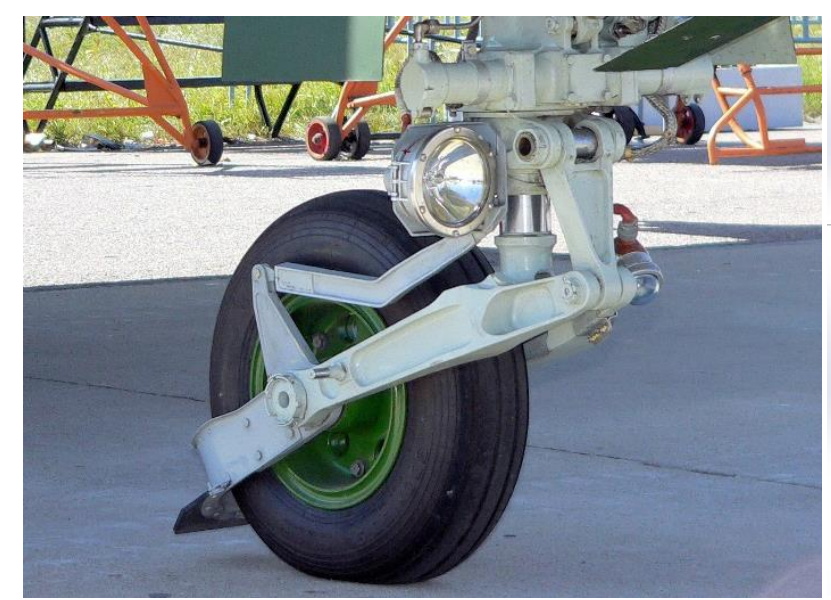

a)

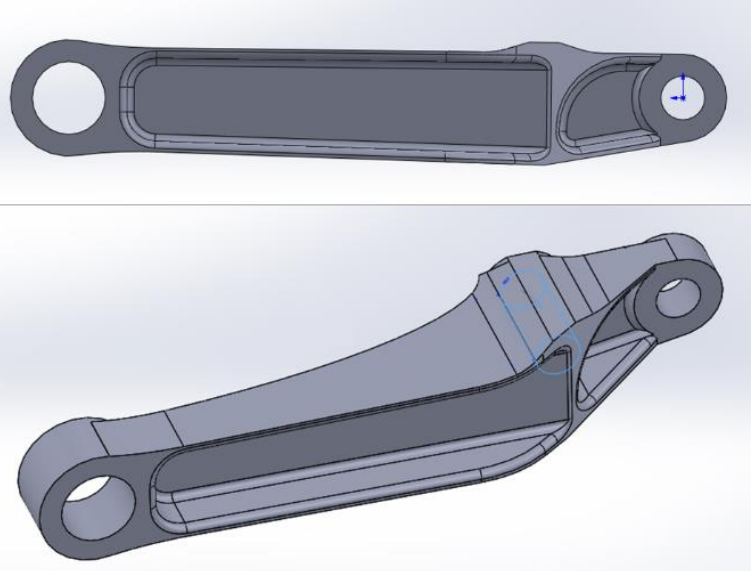

b)

Figure 3: a) photo of the front landing gear of the Yak-130 aircraft, b) 3D model of the fork

When hinged fastening in the bushings of the fork attachment to the rack and bending the impact of the applied force, a flat I-beam bridge works for tension-compression. Its replacement with a fractal lattice under certain conditions can reduce the mass and increase the rigidity of the part. For this reason, experiments were carried out with elements of the fractal lattice applicable in this case.

"The base part" of such an element is a triangle assembled from plates of constant thickness. These plates perceive the main part of the compressive load, which is the reason for this name [10]. A fractal grid of thinner plates is placed inside the base triangle, which prevents the loss of stability of the basic elements. The grid is called "the supporting frame". The optimal ratio of the thickness of the plates of the base elements and the supporting frame at each iteration of the fractal was determined similarly to the method described in [10].

During the experiment, 4 models of the Sierpinski triangle of the zero, first, second and third iterations were studied in the SolidWorks program (Fig. 4, a). The strength limit of the samples printed using these models on a 3D printer was also determined. The height of the triangles was $150 \mathrm{~mm}$, width$20 \mathrm{~mm}$, weight-50 g (Fig. 4, b). The material used is ABS.
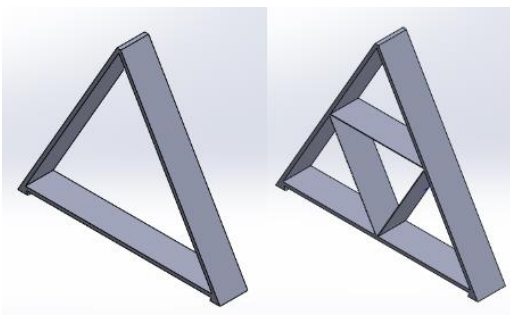

a)
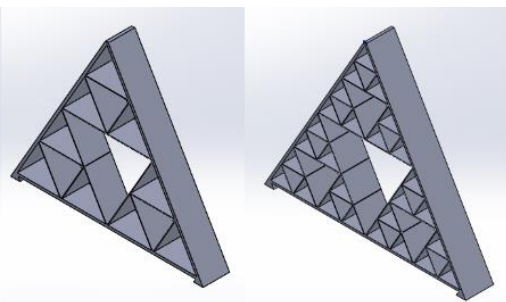

b)

Figure 4: a) 3D models of the Serpinski plate triangle and b) their physical models made of $A B S$

This structure has a clear advantage over ordinary lattices: with the same rigidity, the Sierpinski triangle allows you to save material, leaving voids inside the lattice. The material savings increase exponentially with the growth of the iteration.

Strength studies of the model of the fork of the front landing gear of the Yak-130 military training aircraft were carried out only in the SolidWorks program. At the same time, a number of parametric 
models were used, which made it easier to measure the strengths of parts of different weights. The material used in the calculations is aluminum alloy 7075-T6 (SN). The length of the part is $490 \mathrm{~mm}$, the weight is $2700 \mathrm{~g}$.

\section{Results and discussion}

When interpreting the experimental results, it is important to consider the strength limits of parts in conjunction with buckling, since the use of fractals increases stability by reducing strength. This is clearly demonstrated by the graphs presented in Figure 5.

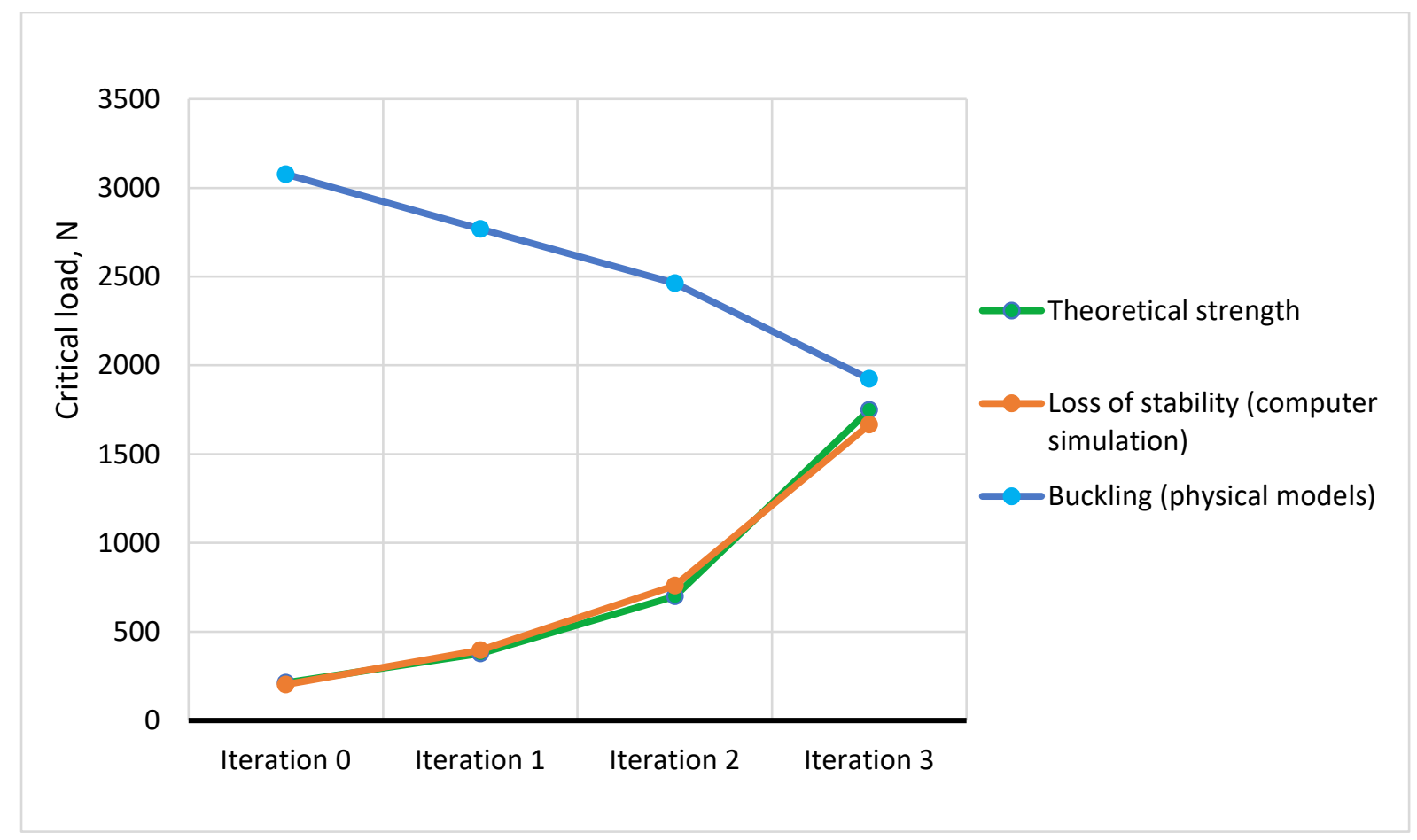

Figure 5: Results of strength studies of Sierpinski triangles

Theoretically, the strength of the material is sufficient for a triangle of plates with a thickness of 3 $\mathrm{mm}$ to withstand a compressive load of more than $3000 \mathrm{~N}$, but both the results of the simulation in SolidWorks and the strength test of physical models (Fig. 6) showed in fact such a triangle will lose stability even with a load of slightly more than $210 \mathrm{~N}$ (Fig. 5).

Therefore, despite the drop in the theoretical strength of the triangle caused by the redistribution of part of the mass from the base elements to the supporting frame, with an increase in the number of iterations, its actual strength increases significantly: in three iterations, it was possible to increase the actual strength of the triangle by more than 8 times.

Extrapolation of the ratios presented in Figure 6 will indicate that under these conditions it is inappropriate to use fractal structures of the fourth and subsequent iterations, since a decrease in the theoretical strength will lead to a decrease in the actual strength. 

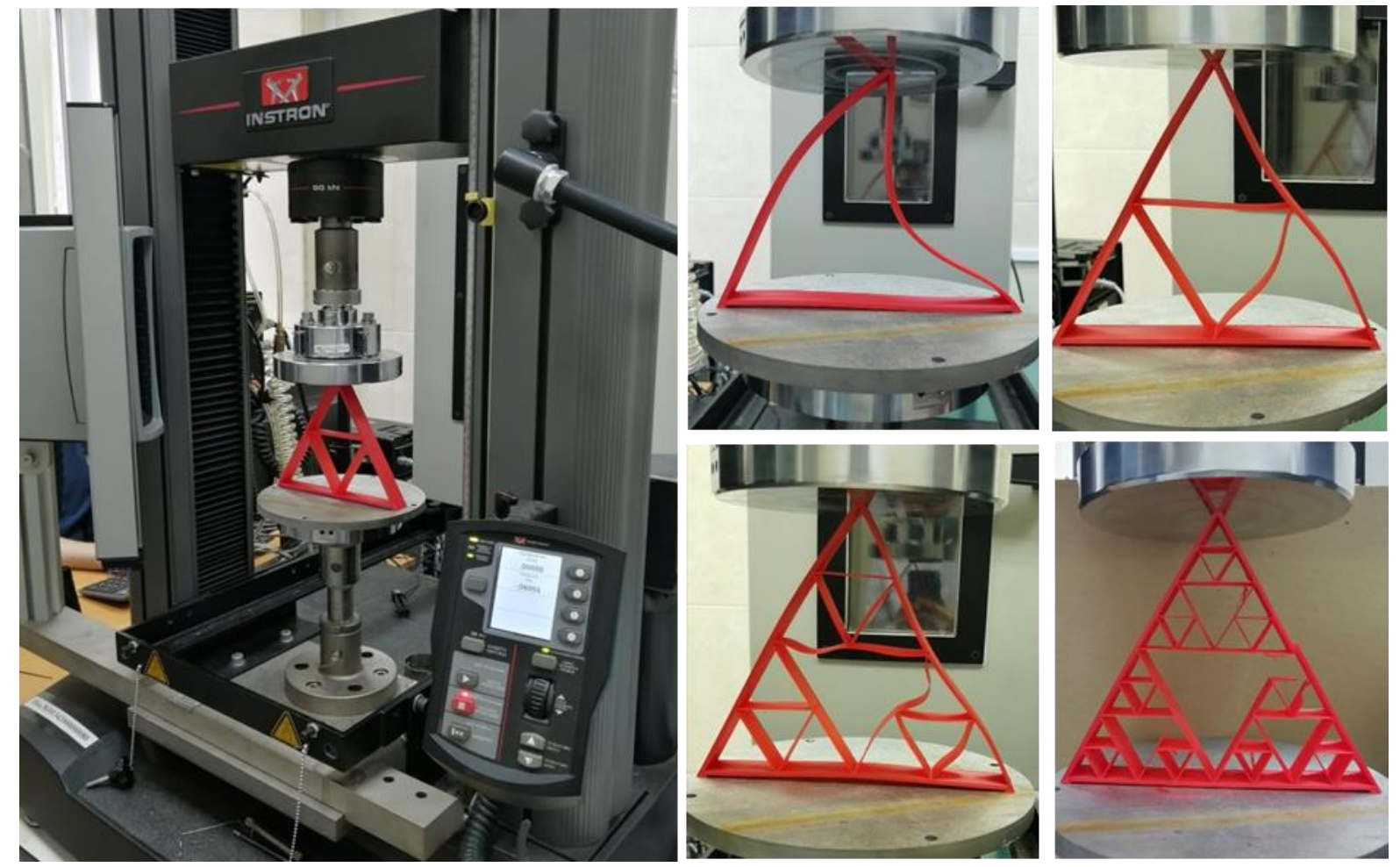

Figure 6: Strength tests of plastic Sierpinski triangle models

The fork of the front chassis of the Yak-130 is a more difficult detail to analyze. The simulation in SolidWorks showed that the stability margin of the part exceeds the safety margin by almost 10 times. To test the effectiveness of using fractal lattices, it was necessary to compare the strength and stability of the part at different masses. For this reason, a simplified model was built, described by a smaller number of parameters related to each other by mathematical dependencies, which made it possible to quickly change the mass of the model, changing several of its basic parameters. Figure 7 shows graphs of the strength and stability of the fork with an I-beam wall thickness from 7 to $0.5 \mathrm{~mm}$ and, accordingly, masses from 2400 to $1000 \mathrm{~g}$.

Such a large minimum mass is explained by the presence of immutable parameters (Fig. 8.). According to the theory, the strength graph is close to a straight line, and the stability graph is close to a parabola. The stability of the fork significantly exceeds its strength over almost the entire mass range under study. Nevertheless, even under such conditions, the use of fractal lattices can be justified: replacing the bridge of I-beam elements with lighter fractals reduces the total mass. However, since the jumper performs not only a supporting function, but also perceives part of the load, the strength also decreases. The ratio of gain in mass and loss in strength is different when using an I-beam (Fig. 8, a), a simple lattice (Fig. 8, b) and a fractal lattice (Fig. 8, c).

Dependencies shown in Figure 9 allow us to estimate these ratios.

The involved dependencies indicate that the lattice of the fractal of the zero iteration reduces the strength of the part at any mass, and the fractals of the first and second iteration increase the strength only at masses less than $1400 \mathrm{~g}$ and $1350 \mathrm{~g}$, respectively. The gain in strength increases with a decrease in weight, although even at the minimum values under consideration does not exceed $10 \%$. Increasing the strength of the part by changing the geometry of its parts allows you to proportionally reduce its weight.

Such a modest efficiency of fractals in solving this problem is explained by the fact that only a small part of the part is replaced by a fractal lattice, which means that even with a significant improvement in strength or mass characteristics, the contribution to the overall specific strength of the part is not so noticeable. 


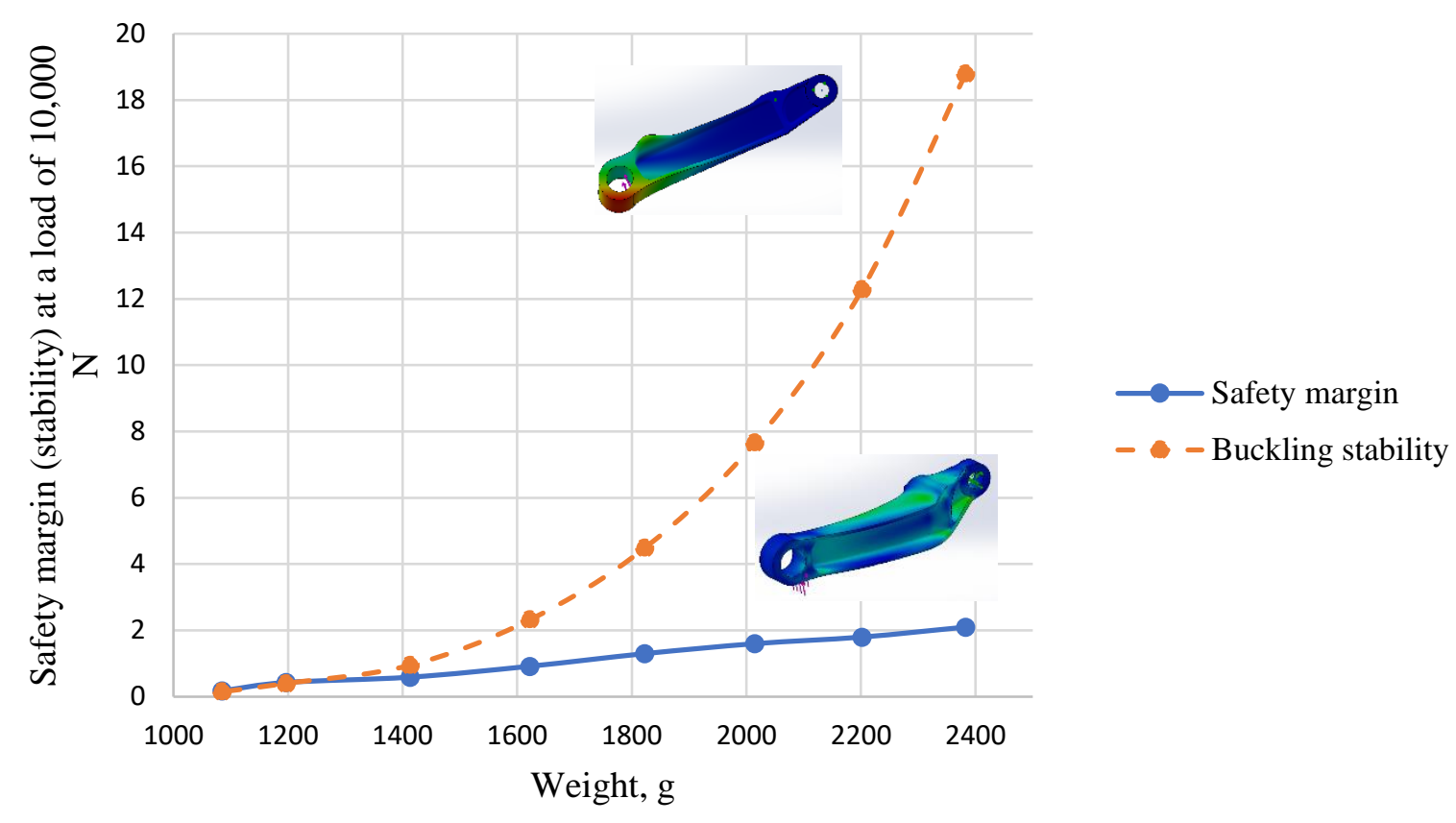

Figure 7: Graphs of comparing the strength and stability of the fork at different weights

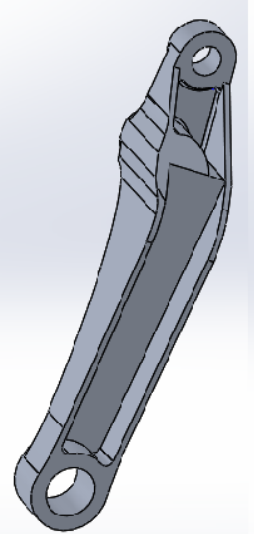

a)

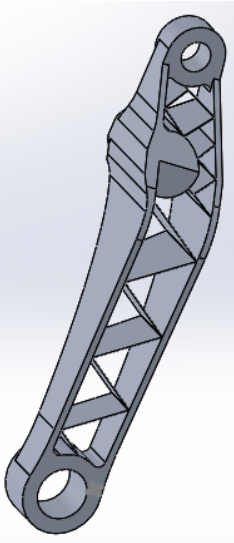

b)

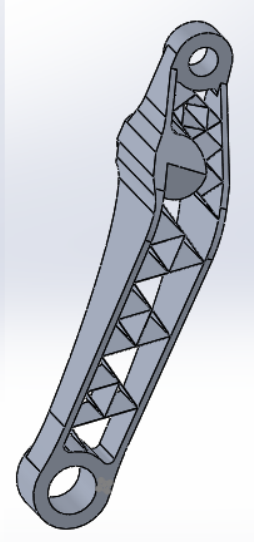

c)

Figure 8: Chassis fork based on -a) I-beam, b) simple lattice and c) fractal lattice (first iteration) 


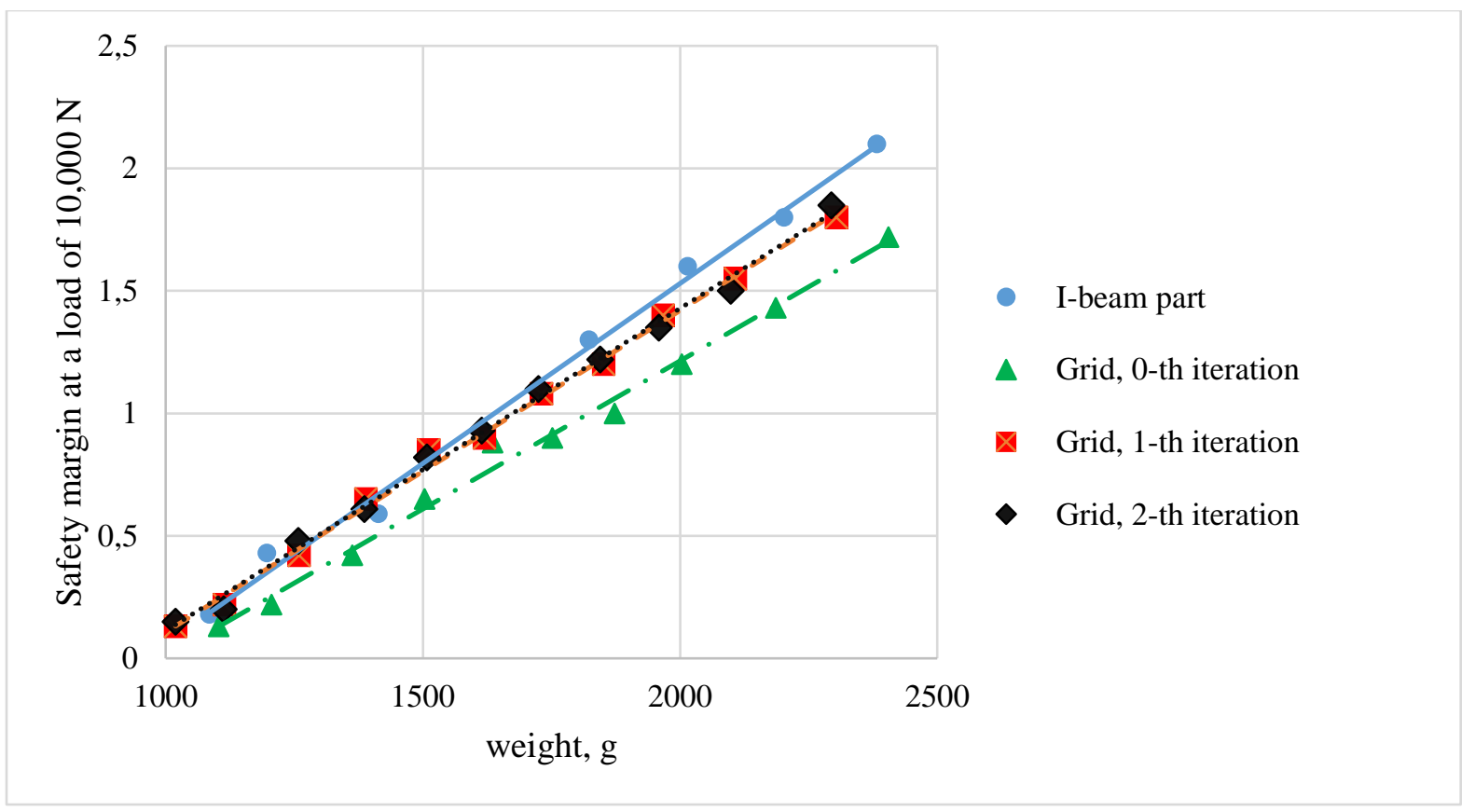

Figure 9: Comparison of the strength of four types of forks at different weights

\section{Summary and conclusion}

In the course of this study, the high efficiency of using the lattice created on the basis of the Sierpinski fractal was confirmed when solving the problem of increasing the stability of the part. The supporting frame increases the rigidity many times, but at the same time the theoretical strength of the part decreases. This can be extremely useful when the filling density of the part volume is low.

The use of fractal gratings to reduce the weight of the fork of the front landing gear of the Yak-130 aircraft has not shown its effectiveness. The use of fractals is justified only with significantly lower operational loads.

According to the author, the details of unmanned aerial vehicles correspond to the above requirements to a much greater extent [12]. The latter are characterized by lower flight speeds and load capacity, which also causes lower loads. It is planned to conduct further research in this area.

\section{Acknowledgements}

The author expresses gratitude to his supervisor, associate professor of the Department of Engineering Graphics of the MAI, Leonid Vladimirovich Markin for his leadership in the study, as well as Arseniy Vladimirovich Babaytsev, a junior researcher at the MAI, for providing access to equipment and carrying out strength studies of physical models of the Serpinsky triangle. The work was performed using the software of the Department of Engineering Graphics of the Russian Technological University MIREA and the Moscow Aviation Institute (National Research University).

\section{References}

[1] K. Sharma, G. Srinivas. Flying smart: Smart materials used in aviation industry, Materials Today: Proceedings 27 (2020): 244-250. doi:10.1016/j.matpr.2019.10.115

[2] I. Meneghin, G. Ivetic, M. Stiller, G. Molinari, V. Ristori, S. Della Ratta, F. Dumont. Fatigue in additive manufactured aircraft: The long way to make it fly, International Committee on Aeronautical Fatigue. Springer, Cham, (2019). doi:10.1007/978-3-030-21503-3_2 
[3] M. Viscardi, M. Arena, P. Cerreta, P. Iaccarino, S. Inserra Imparat. Inserra Imparato Manufacturing and validation of a novel composite component for aircraft main landing gear bay, Journal of Materials Engineering and Performance 28.6 (2019): 3292-3300. DOI: 10.1007 / s11665-019-04106-y

[4] C. Barile, C. Casavola, F. De Cillis. Mechanical comparison of new composite materials for aerospace applications, Composites Part B: Engineering 162 (2019): 122-128. doi:10.1016/j.compositesb.2018.10.101

[5] M. Viscardi, M. Arena, P. Cerreta, P. Iaccarino. Design and prototyping of a novel composite architecture for a widebody landing gear bay, Materials Today: Proceedings 34 (2021): 288-292. doi:10.1016/j.matpr.2020.03.694

[6] C. Hodonou, M. Balazinski, M. Brochu, C. Mascle. Material-design-process selection methodology for aircraft structural components: application to additive vs subtractive manufacturing processes, International Journal of Advanced Manufacturing Technology 103 (2019). DOI: 10.1007 / s00170-019-03613-5

[7] J. Hub. A study on topology optimization of airplane air brake bracing beam, International Conference on Military Technologies (ICMT). IEEE, 2019

[8] D. Rayneau-Kirkhope, Y. Mao, R. Farr, J. Segal. Hierarchical space frames for high mechanical efficiency: Fabrication and mechanical testing, Mechanics Research Communications 46 (2012): 41-46. doi.org/10.1016/j.mechrescom.2012.06.011

[9] D. Rayneau-Kirkhope, Y. Mao, R. Farrb. Ultralight fractal structures from hollow tubes, Physical review letters 109.20 (2012). 204301. DOI:https://doi.org/10.1103/PhysRevLett.109.204301

[10] L. Zhikharev. A Sierpiński triangle geometric algorithm for generating stronger structures, Journal of Physics: Conference Series. Vol. 1901. No. 1. IOP Publishing, (2021). doi:10.1088/17426596/1901/1/012066

[11] A. Dondukov, O. Demchenko, N. Dolzhenkov, A. Matveev, K. Popovich, V. Shkolin, V. Fesenko. Maneuverable training and multi-purpose aircraft "YAK-130". (2000) (in Russian)

[12] C. Courtin, R. John Hansman. M. Drela. Flight Test Results of a Subscale Super-STOL Aircraft. AIAA Scitech 2020 Forum. (2020) 